\title{
CMR assessment of longitudinal left ventricular function following transcatheter aortic valve implantation (TAVI) for severe aortic stenosis
}

\author{
Laura E Dobson ${ }^{1 *}$, Tarique A Musa ${ }^{1}$, Timothy A Fairbairn', Akhlaque Uddin', Daniel J Blackman², David P Ripley ${ }^{1}$, \\ Peter P Swoboda', Adam K McDiarmid', Bara Erhayiem', Pankaj Garg', Sven Plein', John P Greenwood ${ }^{1}$
}

From 18th Annual SCMR Scientific Sessions

Nice, France. 4-7 February 2015

\section{Background}

Global longitudinal strain and mitral annular plane systolic excursion (MAPSE) have been validated as an echocardiographic measure of longitudinal function in patients with aortic stenosis (AS). Cardiac magnetic resonance imaging (CMR) offers a simple and easy measure of MAPSE and been validated in various patient groups. Longitudinal function in aortic stenosis is usually reduced before a reduction in ejection fraction is seen. We sought to investigate the impact of TAVI upon longitudinal function and global ejection fraction in patients treated for symptomatic severe AS.

\section{Methods}

We prospectively enrolled 52 patients with symptomatic severe aortic stenosis undergoing TAVI over a 5 year period from March 2009 to March 2014. Patients with contraindications to CMR were excluded and all patients provided informed written consent. All patients underwent an identical 1.5T CMR protocol (Intera, Phillips Healthcare, Best, The Netherlands) at baseline and at a median of 6 months following TAVR. Multi-slice, multi-phase cine imaging was performed using a standard steady-state free procession pulse sequence in the short and long axis $(8 \mathrm{~mm}$ thickness, $0 \mathrm{~mm}$ gap, 30 phases, typical field of view (FOV) $340 \mathrm{~mm}$ ) to cover the entire left and right ventricle. Longitudinal atrioventricular motion was measured at the septal and lateral mitral annulus in the 4 chamber cine view (Figure 1) at end systole and end diastole. Offline analysis (CVI42, Circle Cardiovascular Imaging, Calgary, Alberta, Canada) included measuring the perpendicular distance between these two points.

\section{Results}

52 patients (mean age $80.3 \pm 6.8$ years, 30 (58\%) male, AVA $0.6 \pm 0.2 \mathrm{~cm}^{2}$, Logistic Euroscore $\left.20.1 \pm 13.9 \%\right)$ were studied. There was no significant change in the overall LV ejection fraction 6 months following TAVI compared to baseline. All three measures of longitudinal function MAPSE $^{\text {Lateral }}$, MAPSE ${ }^{\text {Medial }}$ and MAP$\left.\mathrm{SE}^{\text {Average }}\right)$ were significantly improved 6 months following TAVI (Table 1). There was a moderate correlation between all three MAPSE values and LV ejection fraction (MAPSE ${ }^{\text {lateral }} \mathrm{r}=0.38, \mathrm{p}=0.005$, MAPSE $^{\text {medial }} \mathrm{r}=0.42, \mathrm{p}=0.002$ and MAPSE Average $^{-}$ $r=0.44, p=0.001$ ). Presence or absence of late gadolinium enhancement did not impact on the baseline MAPSE $^{\text {average }}(9.7 \mathrm{~mm}$ Vs $9.1 \mathrm{~mm}, \mathrm{p}=0.44)$ or have impact on the change in MAPSE ${ }^{\text {average }}$ following TAVI $(0.7 \mathrm{~mm}$ Vs $1.3 \mathrm{~mm}, \mathrm{p}=0.48)$.

\section{Conclusions}

Longitudinal left ventricular function, as assessed using MAPSE, improved following TAVI for severe aortic stenosis. MAPSE may be a more sensitive marker of left ventricular recovery following TAVI than global LV ejection fraction.

\section{Funding}

This study was part funded by the British Heart Foundation (PG/11/126/29321). 


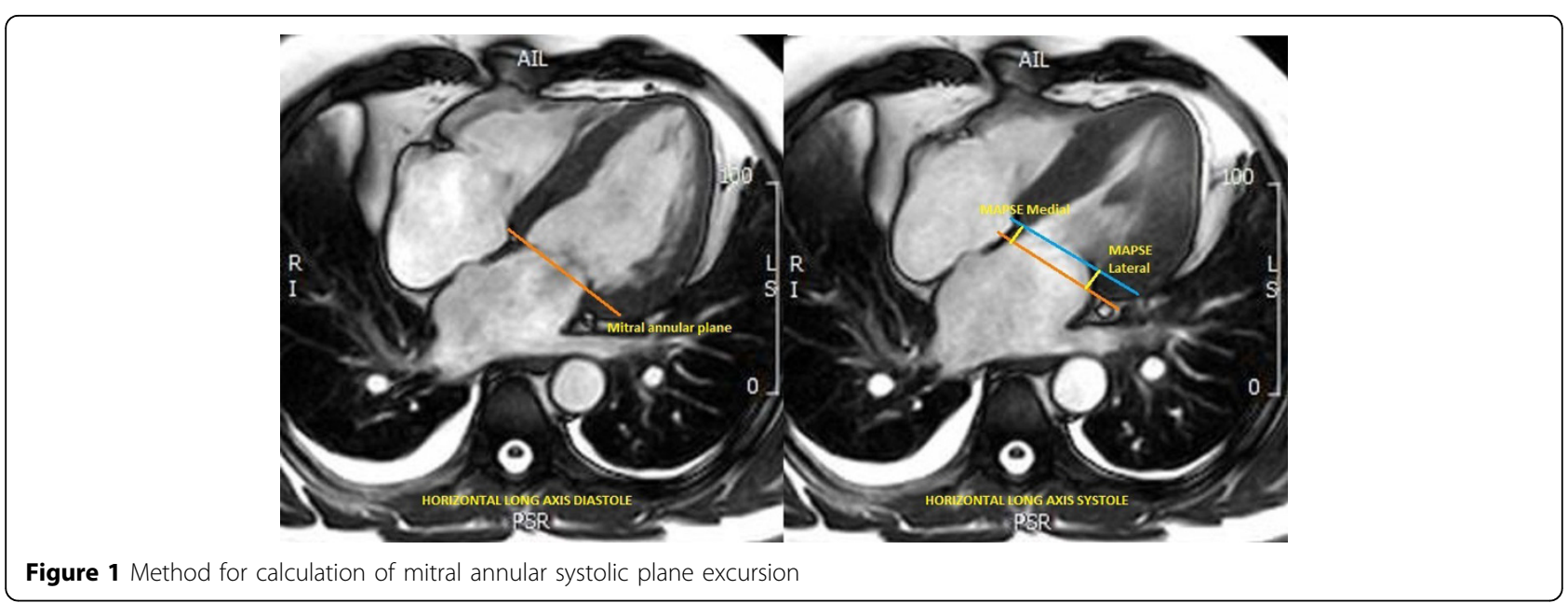

Table 1 Mean LV ejection fraction and MAPSE values before and 6 months after TAVI. Values expressed as mean \pm SD.

\begin{tabular}{cccc}
\hline & Pre-TAVI & Post-TAVI & P Value \\
\hline LV ejection fraction, $\%$ & $54.9 \pm 13.2$ & $56.8 \pm 11.9$ & 0.08 \\
\hline MAPSELateral, $\mathrm{mm}$ & $10.6 \pm 2.9$ & $11.7 \pm 3.1$ & 0.005 \\
\hline MAPSEMedial, $\mathrm{mm}$ & $8.5 \pm 2.4$ & $9.2 \pm 2.4$ & 0.04 \\
\hline MAPSEAverage((medial + lateral) / 2), $\mathrm{mm}$ & $9.6 \pm 2.4$ & $10.5 \pm 2.6$ & 0.005 \\
\hline
\end{tabular}

\section{Authors' details}

${ }^{1}$ Multidisciplinary Cardiovascular Research Centre \& Leeds Institute for

Cardiovascular and Metabolic Medicine, University of Leeds, Leeds, UK.

${ }^{2}$ Yorkshire Heart Centre, Leeds Teaching Hospitals Trust, Leeds, UK.

Published: 3 February 2015

doi:10.1186/1532-429X-17-S1-P180

Cite this article as: Dobson et al: CMR assessment of longitudinal left ventricular function following transcatheter aortic valve implantation (TAVI) for severe aortic stenosis. Journal of Cardiovascular Magnetic

Resonance 2015 17(Suppl 1):P180.

Submit your next manuscript to BioMed Central and take full advantage of:

- Convenient online submission

- Thorough peer review

- No space constraints or color figure charges

- Immediate publication on acceptance

- Inclusion in PubMed, CAS, Scopus and Google Scholar

- Research which is freely available for redistribution 